\title{
Does Improved Attention Induced by Caffeine Intake Affect Olfactory Function?
}

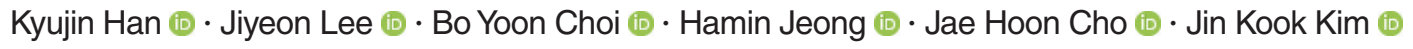 \\ Department of Otorhinolaryngology-Head and Neck Surgery, Konkuk University School of Medicine, Seoul, Korea
}

Objectives. Past several studies have proven that caffeine facilitates attentional enhancement by acting as an adenosine antagonist once it is absorbed by the body, resulting in improved psycho-behavioral function. Modern clinical olfactory function tests are usually assessed by psychophysical tests but due to a paucity of data, the influence of enhanced attention by caffeine on olfactory function still remains unclear. The objective of this study was to compare results of cognitive function (attention) and olfactory function before and after caffeine administration in order to analyze effects of caffeine on olfactory function in normosmic subjects.

Methods. This study enrolled 49 participants of Konkuk University Hospital with a mean age of 27.7 years who had patent olfactory clefts and no olfactory dysfunction from May 2015 to February 2016. Subjects were restrained from caffeine 10 hours before the test. On day 1, participant's subjective olfactory function was evaluated before and after uptake of either caffeinated or decaffeinated coffee using visual analog scale (VAS) score, minimum cross-sectional area (MCA) measured by acoustic rhinometry, and the Korean version of Sniffin' Stick II (KVSS II). Evaluation of participant's attentional degree was measured by $\mathrm{d} 2$ test. On day 2 , the same procedure was carried out with counterpart substance. The type of coffee initially administrated was randomly selected.

Results. After administration, caffeinated coffee resulted in significant attentional enhancement than decaffeinated coffee. Results of $\mathrm{d} 2$ test showed statistically significant differences in the parameters of total number of errors and omission errors. In both the caffeinated and decaffeinated groups, the patients showed slight increase in VAS score and nasal cavity area; however, the difference was not statistically significant. Also, caffeinated coffee intake compared to decaffeinated coffee intake showed no significant relevance to olfactory function.

Conclusion. Caffeine may significantly improve attentional congnitive function, while not have acute effects on olfactory function.

Keywords. Caffeine; Attention; Smell

\section{INTRODUCTION}

Caffeine is one of the most extensively consumed psychoactive substances worldwide. It is concentrated in various forms of beverages, including coffee, tea, and soft drinks [1,2]. Complex

\footnotetext{
- Received October 4, 2018

Revised December 4, 2018

Accepted December 20, 2018

- Corresponding author: Jin Kook Kim

Department of Otorhinolaryngology-Head and Neck Surgery, Konkuk

University School of Medicine, 120-1 Neungdong-ro, Gwangjin-gu, Seoul 05030, Korea

Tel: +82-2-2030-7662, Fax: +82-2-2030-5299

E-mail: entalk@kuh.ac.kr
}

behavioral and biochemical effects can be triggered extensively by caffeine throughout the human body [3]. Accordingly, many studies have focused on effects of caffeine on central nervous system (CNS) and cognitive processes [4-8]. Wentz and Magavi [9] have reported that extended administration of supraphysiological doses of caffeine has several biochemical effects. It can alter the activity of cortical, thalamic, and striatal neurons. Drinking coffee can also enhance dynamic cerebral autoregulation [10]. Other researches have explored acute effects of tea on cognitive functions, particularly attention [11,12]. As a stimulant, caffeine often increases alertness and improves performance for tasks requiring sustained attention [13]. In addition, it was reported that acute intake of theanine, caffeine, and their

Copyright $\odot 2020$ by Korean Society of Otorhinolaryngology-Head and Neck Surgery.

This is an open-access article distributed under the terms of the Creative Commons Attribution Non-Commercial License (https://creativecommons.org/licenses/by-nc/4.0)

which permits unrestricted non-commercial use, distribution, and reproduction in any medium, provided the original work is properly cited. 
significantly improved cognitive and neurophysiological measures of selective attention [14].

In terms of sense of smell, previous animal studies have indicated that administration of caffeine can improve olfactory function [15-17]. Attentional enhancement of caffeine is attributed to its inhibition of adenosine receptors (types A1 and A2a) which in turn can increase dopaminergic and cholinergic transmission in the brain. It has been found that adenosine-30,50-cyclic monophosphate is increased in various brain areas which can explain central nervous effects of methylxanthines such as theophylline and caffeine $[18,19]$. Hence, olfaction is not only affected by anatomical state of nasal epithelium, but also affected by the CNS and peripheral nervous system. However, it has been previously reported by Meusel et al. [20] that short-term administration of caffeine has little or no effect on patients with post-upper respiratory infection or sinonasal olfactory dysfunction. Also, it was mentioned in the report that the effects of caffeine on olfactory function, especially on normosmic subjects, have not been tested and in such circumstances, improvement on odor discrimination as compared to odor thresholds would be expected.

From our past clinical experience, olfactory dysfunction patients raised question of whether lack of attention and concentration after time-consuming olfactory function test had any relation to their poor scores. Therefore, it is meaningful to examine whether attentional enhancement induced by caffeine uptake has effect on olfactory function. In this study, we compared results of olfactory function test and cognitive function (attention) before and after caffeine administration to analyze effects of caffeine on subjects with normal olfactory function. In addition, we evaluated the relationship between improvement of cognitive function and Korean version of Sniffin' Stick II (KVSS II) test results.

\section{MATERIALS AND METHODS}

\section{Study population}

This study included 49 consecutive healthy participants (34 males and 15 females) who volunteered at the Department of Otorhi-

\section{H I G H L I G H T S}

- Acute caffeine intake resulted in significant attentional enhancement compared to the decaffeinated drink intake in the d2 test.

- No significant difference was noticed in the increase of nasal cavity area after caffeine intake or improvement of olfactory function in normosmic subjects.

- Enhanced cognition induced by caffeine administration may not have acute effects on olfactory function.
nolaryngology-Head and Neck surgery, Konkuk University Medical Center, from April 2015 to May 2016. We excluded participants with symptoms or signs suggesting other sinonasal diseases or disorders of the CNS. Thus, participants with obstructed olfactory clefts or other diseases entities that might cause conductional olfactory dysfunction such as nasal septal deviation or nasal polyps were excluded. Those with a history of posttraumatic and post-infectious olfactory dysfunction or other miscellaneous types of olfactory disorder with decreased sense of smell were also excluded. All participants were fully notified of the contents of this study. They agreed to participate after obtaining informed consent under approval of Institutional Review Board (IRB No. KUH1110063).

\section{Study design}

This study was a double-blinded clinical trial. Subjects underwent a series of test for 2 days. Considering the half-life of caffeine which is known to be 4-5 hours with modest intake [21], each subject was restrained from caffeine consumption at an extended period of 10 hours for sufficient clearance. On the first day, individuals were tested for their olfactory visual analog scale (VAS) score, minimum cross-sectional area (MCA) measured by acoustic rhinometry, KVSS II and $\mathrm{d} 2$ test prior to coffee consumption. These same tests were then conducted $30 \mathrm{~min}$ utes after drinking a cup of coffee containing $72 \mathrm{mg}$ of caffeine or decaffeinated coffee with $8.4 \mathrm{mg}$ caffeine (KANU, dark roast Americano or KANU, decaffeine Americano; Dong Suh Food Co., Seoul, Korea). On the second day, the same experiment was done. However, each individual was asked to drink the opposite type of coffee as they did the day before. The type of coffee initially administered was randomly selected. Results of the olfactory function and $\mathrm{d} 2$ test before and after caffeinated and decaffeinated coffee intake were analyzed respectively to determine statistical significance. Because neuropsychological tests are affected by circadian rhythm, all subjects were tested at 17:00 PM consistently.

\section{Evaluation of olfactory function}

A survey was conducted to evaluate subjects using VAS. Results of acoustic rhinometry MCA was measured. All participants were initially examined by an otorhinolaryngologist (KJK) with a $2.7 \mathrm{~mm} \times 0^{\circ}$ nasal endoscope to check the patency of the olfactory cleft, the existence of nasal polyps or nasal obstruction due to septal deviation, mucoid or purulent discharge, and any other signs of sinonasal diseases. Olfactory function was evaluated with KVSS II that offers a comprehensive measurement of individual's olfactory function. It consists of three different sets that measures T (olfactory threshold), D (discrimination), and I (identification). These tests are performed in the same way as the Sniffin' Stick test. Threshold was defined as the concentration where n-butanol (highest concentration 4\%, 1:2 serial dilutions to 16 steps) was correctly identified four times in a row 
from two blanks. For discrimination, triplets of odorants (two of them are identical, one is different) are presented and subjects have to choose the different one. In the identification test, subjects were asked to choose from 16 items that was recorded with as score ranging from 0 to 16 . Theses scores were subsequently added up as the "KVSS II total score (TDI score)." Total scores of 0-20, 20-27, and 28-48 were defined as "anosmia," "hyposmia," and "normosmia," respectively $[22,23]$.

\section{Evaluation of attention}

Evaluation of participant's attention was measured with the $\mathrm{d} 2$ test, developed by Brickenkamp and Zillmer [24]. The $\mathrm{d} 2$ test measures attention with a time-limit. By differentiating similar optical stimulus, values such as processing-speed, regulation obedience, and quality of performance can be measured. It is a tool that evaluates an individual's attention and concentration ability. The $\mathrm{d} 2$ test is composed of 658 stimulants, each of which is categorized into 16 different subtypes. For instance, alphabet "d" or "p" are marked with one to four dashes and subject is required to select and respond to only d's with two dashes (d"). The d2 test is composed of several different parameters. For instance, the total number of items processed measures processing speed, while the total number-total error (TN-E), which is the number of correctly marked characters minus the number of incorrectly marked, evaluates overall performance. Concentration performance, which is measured by total number of correctly cancelled minus total number, also represents overall performance. Fluctuation rate is a measure of consistency and stability of the performer.

Table 1. Demographics and descriptive statistics of the study population

\begin{tabular}{lc}
\hline Characteristics & Value $(\mathrm{n}=49)$ \\
\hline Age $(\mathrm{yr})$ & $27.7 \pm 2.8$ \\
Female:male & $15: 34$ \\
VAS & $7.9 \pm 1.2$ \\
MCA, right & $0.6 \pm 0.3$ \\
MCA, left & $0.6 \pm 0.3$ \\
KVSS II test (TDI score) & $29.0 \pm 4.8$ \\
Threshold score & $6.3 \pm 2.5$ \\
Discrimination score & $10.5 \pm 2.3$ \\
Identification score & $12.1 \pm 2.1$ \\
d2 test & \\
Total no. of items processed & $608.8 \pm 57.3$ \\
Omission error & $17.6 \pm 22.5$ \\
Commission error & $1.2 \pm 3.2$ \\
Total no. of error & $17.8 \pm 22.7$ \\
TN-E & $590.9 \pm 68.6$ \\
Concentration performance & $249.3 \pm 40.7$ \\
Fluctuation rate & $7.6 \pm 6.9$ \\
\hline
\end{tabular}

Values are presented as mean \pm standard deviation.

VAS, visual analog scale; MCA, minimum cross-sectional area; KVSS II, Korean version of Sniffin' Stick II; TDI, threshold, discrimination, identification; TN-E, total number-total error.

\section{Statistical analysis}

Statistical analyses were performed using SPSS ver. 17.0 (SPSS Inc., Chicago, IL, USA). To determine differences in VAS score, acoustic rhinometry, KVSS II, and $\mathrm{d} 2$ test between results of before and after caffeinated and decaffeinated coffee intake, continuous variables were tested with paired $t$-test. A 2 -tailed $P$-value of less than 0.05 was considered statistically significant.

\section{RESULTS}

\section{Demographics of participants}

This study was carried on 49 patients (34 males and 15 females) with a mean age of $27.7 \pm 2.8$ years (range, 24 to 35 years). The average subjectiveVAS score of participants was $7.9 \pm 1.2$. Results of the acoustic rhinometry MCA without any administration of caffeine or decaffeinated drink showed an average of $0.6 \pm 0.3 \mathrm{~cm}^{2}$ on the right side of the nasal cavity and $0.6 \pm 0.3 \mathrm{~cm}^{2}$ on the left side. The mean KVSS II total score was $29.0 \pm 4.8$. Average TDI score was threshold, $6.3 \pm 2.5$; discrimination, $10.5 \pm 2.3$; and identification, $12.1 \pm 2.1$. In $\mathrm{d} 2$ test, the following results were obtained: mean score of total number of items processed, $608.8 \pm$ 57.3; omission error, $17.6 \pm 22.5$; commission error, $1.2 \pm 3.3$;

Table 2. Comparison of VAS score and MCA before and after caffeinated or decaffeinated coffee intake

\begin{tabular}{lccc}
\hline Variable & Caffeinated & Decaffeinated & $P$-value* \\
\hline VAS & $0.4 \pm 1.0$ & $0.2 \pm 1.3$ & 0.2 \\
MCA, right & $0.1 \pm 0.6$ & $0.0 \pm 0.5$ & 0.4 \\
MCA, left & $0.1 \pm 0.5$ & $-0.1 \pm 0.4$ & 0.8 \\
\hline
\end{tabular}

Values are presented as mean \pm standard deviation.

VAS, visual analog scale; MCA, minimum cross-sectional area. ${ }^{*} P<0.05$.

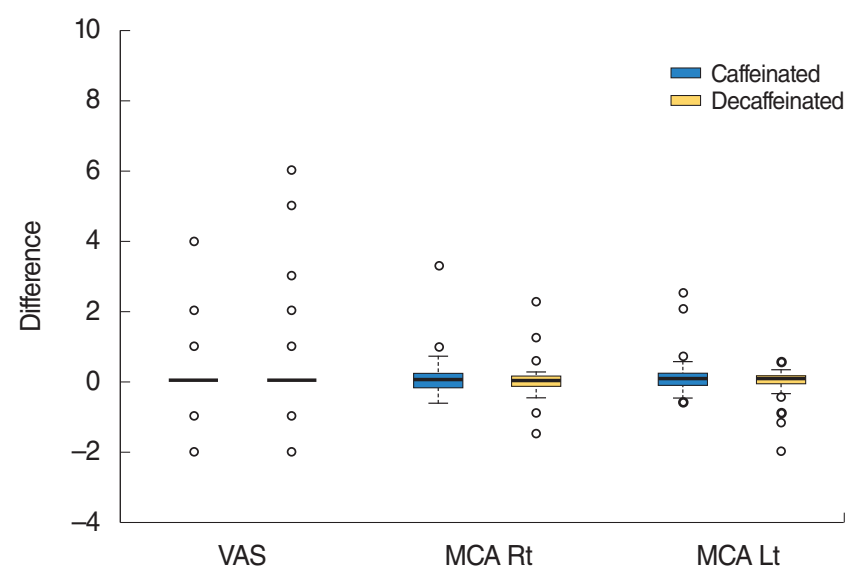

Fig. 1. Comparison of the difference between the mean visual analog scale (VAS) score and minimum cross-sectional area (MCA) before and after caffeinated or decaffeinated coffee intake. Results showed no significant increase of VAS score or MCA $(P>0.05)$. Rt, right; Lt, left. 
Table 3. Comparison of KVSS II test score before and after caffeinated or decaffeinated coffee intake

\begin{tabular}{lccc}
\hline Variable & Caffeinated & Decaffeinated & $P$-value* \\
\hline KVSS II test & $1.0 \pm 3.8$ & $0.3 \pm 3.0$ & 0.2 \\
Threshold score & $0.2 \pm 2.6$ & $0.1 \pm 2.2$ & 0.8 \\
Discrimination score & $0.4 \pm 2.2$ & $0.1 \pm 1.7$ & 0.4 \\
Identification score & $0.0 \pm 1.9$ & $0.2 \pm 1.2$ & 0.7 \\
\hline
\end{tabular}

Values are presented as mean \pm standard deviation.

KVSS II, Korean version of Sniffin' Stick II.

${ }^{*} P<0.05$.

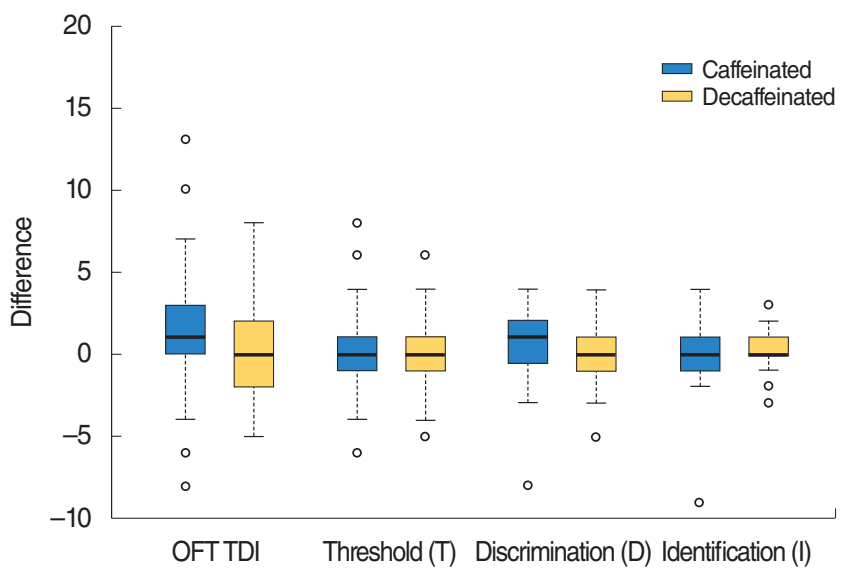

Fig. 2. Comparison of Korean version of Sniffin' Stick II score before and after caffeinated or decaffeinated coffee intake. Subjects showed no significant improvement of olfactory function in threshold, discrimination and identification scores $(P>0.05)$. Lines for the 95\% confidence interval are shown. OFT, olfactory function test.

total number of errors, $17.8 \pm 22.7$; TN-E, $590.90 \pm 68.64$; concentration performances, $249.3 \pm 40.7$; fluctuation rate, $7.6 \pm 6.9$ (Table 1).

\section{Comparison of VAS score, MCA, and KVSS II score before and after caffeinated or decaffeinated coffee intake}

Although the VAS score and nasal cavity area showed slight increase after caffeine intake, there was no statistically significant difference in VAS scores between before and after caffeinated coffee intake or between before and after administration of decaffeinated coffee $(P=0.19$, paired $t$-test). Acoustic rhinometry showed no significant difference in MCAs between right and left sides $(P=0.35)$ (Table 2, Fig. 1). Subjects showed no significant improvement of olfactory function following caffeine administration. Results did not show any significant difference in KVSS II total score, odor threshold, odor discrimination, or odor identification between before and after caffeinated or decaffeinated coffee intake (KVSS II total score, $P=0.19$; threshold score, $P=0.79$; discrimination score, $P=0.35$; identification score, $P=0.69$ ) (Table 3, Fig. 2).
Table 4. Comparison of d2 test before and after caffeinated or decaffeinated coffee intake

\begin{tabular}{lccc}
\hline Variable & Caffeinated & Decaffeinated & $P$-value \\
\hline Total no. of items processed & $19.0 \pm 33.2$ & $20.5 \pm 33.5$ & 0.8 \\
Omission error & $-10.0 \pm 13.1$ & $-4.2 \pm 9.6$ & 0.0 \\
Commission error & $-0.8 \pm 3.4$ & $5.8 \pm 15.5$ & 0.1 \\
Total no. of error & $-9.8 \pm 13.2$ & $-3.9 \pm 10.0$ & 0.0 \\
TN-E & $28.7 \pm 36.1$ & $25.3 \pm 33.6$ & 0.2 \\
Concentration performance & $23.1 \pm 20.2$ & $26.1 \pm 58.7$ & 0.9 \\
Fluctuation rate & $-2.7 \pm 3.9$ & $-2.5 \pm 4.6$ & 0.5 \\
\hline
\end{tabular}

TN-E, total number-total error. ${ }^{*} P<0.05$

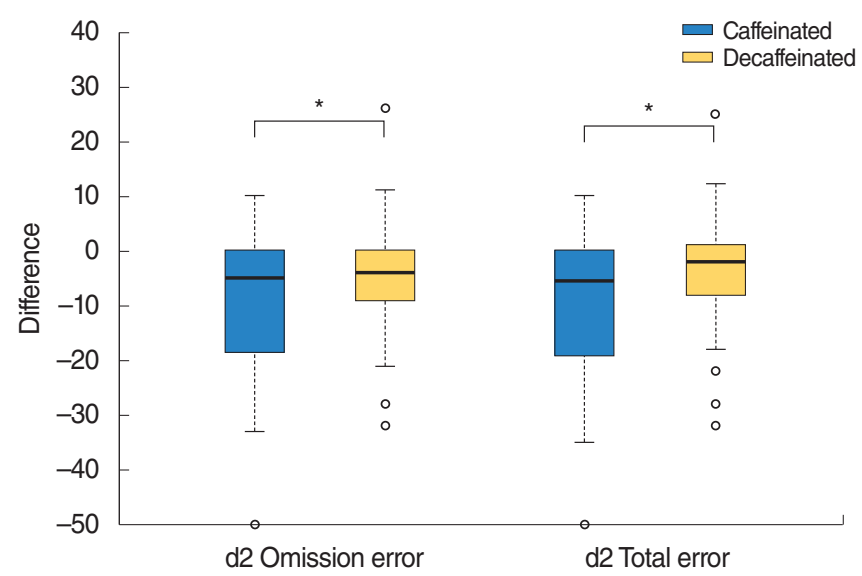

Fig. 3. Comparison of d2 test before and after caffeinated or decaffeinated coffee intake. Values are difference of mean \pm standard deviation. ${ }^{\star} P<0.05$, caffeinated vs. decaffeinated. Lines for the $95 \%$ confidence interval are shown.

\section{Comparison of $\mathrm{d} 2$ test before and after caffeinated or decaffeinated coffee intake}

Results of $\mathrm{d} 2$ test showed statistically significant differences in the parameters of total number of errors and omission errors (total error, $P=0.01$; omission error, $P=0.01$ ). After caffeine intake, the amount of omission error was decreased to $-10.0 \pm$ 13.1 than that before intake. After decaffeinated coffee intake, omission error decrease was $-4.2 \pm 9.6(P=0.01)$. The total number of errors after caffeine intake was decreased to $-9.8 \pm 13.2$ compared to that before intake. After decaffeinated coffee intake, the total number of errors decrease was $-3.9 \pm 10.0(P=$ 0.01 ). There was no significant difference in the remainder parameters of $\mathrm{d} 2$ test (total number of items processed, $P=0.82$; commission error, $P=0.10$; TN-E, $P=0.24$; concentration performances, $P=0.89$; and fluctuation rate, $P=0.49$ ) (Table 4, Fig. 3).

\section{DISCUSSION}

Based on results of the present study, parameters (total number of errors and omission error) of d2 test showed significant de- 
creases after caffeine consumption. This implies that caffeine does have additive effects on cognitive function, consistent with other previous studies including the study of Renda et al. [25] showing that caffeine has a general positive impact on attention. For tasks involving alerting, orienting, and (verbal) executive control, caffeine can positively affect different aspects of attention.

In addition to positive effects of caffeine on attention, we also determined if enhancement of attention might have additive effect on olfaction. However, we did not find any significant relation between caffeine and its effect on olfactory function. There was no significant change in results of MCA or KVSS II score before and after caffeine intake. These results are similar to those of Meusel et al. [20]. Of all 76 hyposmic participants in comparison with placebo, there was no significant effect of caffeine (65 mg/cup) on olfactory function regardless whether it was caused by an acute infection of upper respiratory tract or sinonasal disease. This implies that caffeine has little or no short-term effect on olfactory function in patients with olfactory loss. In comparison to Meusel et al. [20], in our study the amount of caffeine administered and the character of the participants may be comparable influential factors. The amount of caffeine administered was slightly higher which was $72 \mathrm{mg} / \mathrm{cup}$. In addition, participants enrolled for this study were healthy normosmic subjects with normal olfactory function instead of patients with olfactory dysfunction.

There have been studies on the relationship between enhanced attention on other sensory modalities such as hearing and vision. Goel et al. [26] have found that participants who consumed energy drinks containing $80 \mathrm{mg}$ of caffeine show significantly reduced auditory reaction time. However, in other animal studies conducted by Mujica-Mota et al. [27] and Zawawi et al. [28], caffeine might have detrimental effects on hearing recovery after acoustic trauma in guinea pigs. In the case of olfaction, it has been shown that attention to odors can decrease the response time to odors $[29,30]$. Hedner et al. [31] have determined cognitive correlates of olfactory performance. Their results suggested that individual cognitive profile might exert influence on performance in higher order olfactory tasks. Together, these data suggest that it is possible to attend to olfaction in the same way as we may attend to vision or audition. However, further research is needed on this issue.

Previous studies have investigated other substances that can alter olfactory function such as ethanol. For instance, Engen [32] have reported that subjects with ethanol-intoxicated blood levels tend to show enhanced performance for odor detection of guaiacol. However, in another study conducted by Patel et al. [33], acute alcohol ingestion markedly decreased olfactory threshold sensitivity to ethanol, but not to phenyl ethyl alcohol. Thus, the selection of odorant and degree of olfactory dysfunction in subjects should be considered in future studies.

Our study has several limitations. Because participants already had normosmic olfactory function with higher scores in the olfactory function test, the results after caffeine intake may have not shown significant improvement. Also, the efficacy of caffeine which includes administrated dose and the timing of the test which took place around the peak arousal effects of caffeine, known to be 25-30 minutes after ingestion, maybe questioned when relating to olfactory function [5]. Furthermore, owing to the short period of examination after administration, the study cannot determine the effects of extended and accumulated doses of caffeine.

Results of this study indicate that caffeine does have acute additive effects on cognitive function by improving attention. Nevertheless, our data do not support effects of improved attention on olfactory function alteration in normosmic subjects. To investigate the potential effect of caffeine on olfactory function, further studies with extended periods of time and a wider range of administrated doses of caffeine are needed.

\section{CONFLICT OF INTEREST}

No potential conflict of interest relevant to this article was reported.

\section{ACKNOWLEDGMENTS}

This research was supported by Basic Science Research Program through the National Research Foundation of Korea (NRF) funded by the Ministry of Education (NRF-2016R1D1A1B01012705, 2017R1D1A1B03028011), Republic of Korea.

\section{ORCID}

Kyujin Han https://orcid.org/0000-0003-4073-5602

Jiyeon Lee https://orcid.org/0000-0002-3427-7863

Bo Yoon Choi https://orcid.org/0000-0002-8511-7966

Hamin Jeong https://orcid.org/0000-0003-2667-0726

Jae Hoon Cho https://orcid.org/0000-0002-2243-7428

Jin Kook Kim https://orcid.org/0000-0003-4245-6252

\section{AUTHOR CONTRIBUTIONS}

Conceptualization: KJK. Data curation: LJY. Formal analysis: CBY. Funding acquisition: KJK. Methodology: KJK. Project administration: CJH. Visualization: JHM. Writing - original draft: HKJ.Writing - review \& editing: HKJ.

\section{REFERENCES}

1. Gunja N, Brown JA. Energy drinks: health risks and toxicity. Med J Aust. 2012 Jan;196(1):46-9. 
2. Heatherley SV, Hancock KM, Rogers PJ. Psychostimulant and other effects of caffeine in 9- to 11-year-old children. J Child Psychol Psychiatry. 2006 Feb;47(2):135-42.

3. Fredholm BB, Battig K, Holmen J, Nehlig A, Zvartau EE. Actions of caffeine in the brain with special reference to factors that contribute to its widespread use. Pharmacol Rev. 1999 Mar;51(1):83-133.

4. Adan A, Serra-Grabulosa JM. Effects of caffeine and glucose, alone and combined, on cognitive performance. Hum Psychopharmacol. 2010 Jun-Jul;25(4):310-7.

5. Barry RJ, Clarke AR, Johnstone SJ, Rushby JA. Timing of caffeine's impact on autonomic and central nervous system measures: clarification of arousal effects. Biol Psychol. 2008 Mar;77(3):304-16.

6. Barry RJ, Rushby JA, Wallace MJ, Clarke AR, Johnstone SJ, Zlojutro I. Caffeine effects on resting-state arousal. Clin Neurophysiol. 2005 Nov;116(11):2693-700.

7. Serra-Grabulosa JM,Adan A, Falcon C, Bargallo N. Glucose and caffeine effects on sustained attention: an exploratory fMRI study. Hum Psychopharmacol. 2010 Nov;25(7-8):543-52.

8. Snel J, Lorist MM. Effects of caffeine on sleep and cognition. Prog Brain Res. 2011;190:105-17.

9. Wentz CT, Magavi SS. Caffeine alters proliferation of neuronal precursors in the adult hippocampus. Neuropharmacology. 2009 MayJun;56(6-7):994-1000.

10. Sasaki H, Hirasawa A, WashioT, Ogoh S. Acute effect of coffee drinking on dynamic cerebral autoregulation. Eur J Appl Physiol. 2016 May;116(5):879-84.

11. Camfield DA, Stough C, Farrimond J, Scholey AB. Acute effects of tea constituents L-theanine, caffeine, and epigallocatechin gallate on cognitive function and mood: a systematic review and meta-analysis. Nutr Rev. 2014 Aug;72(8):507-22.

12. Einother SJ, Martens VE. Acute effects of tea consumption on attention and mood.Am J Clin Nutr. 2013 Dec;98(6 Suppl):1700S-1708S.

13. Brice C, Smith A. The effects of caffeine on simulated driving, subjective alertness and sustained attention. Hum Psychopharmacol. 2001 Oct;16(7):523-31.

14. Kahathuduwa CN, Dassanayake TL, Amarakoon AM, Weerasinghe VS. Acute effects of theanine, caffeine and theanine-caffeine combination on attention. Nutr Neurosci. 2017 Jul;20(6):369-77.

15. Prediger RD, Batista LC, Takahashi RN. Caffeine reverses age-related deficits in olfactory discrimination and social recognition memory in rats: involvement of adenosine $\mathrm{A} 1$ and $\mathrm{A} 2 \mathrm{~A}$ receptors. Neurobiol Aging. 2005 Jun;26(6):957-64.

16. Si A, Zhang SW, Maleszka R. Effects of caffeine on olfactory and visual learning in the honey bee (Apis mellifera). Pharmacol Biochem Behav. 2005 Dec;82(4):664-72.

17. Siderowf A, Jennings D, Connolly J, Doty RL, Marek K, Stern MB. Risk factors for Parkinson's disease and impaired olfaction in relatives of patients with Parkinson's disease. Mov Disord. 2007 Nov; 22(15):2249-55.
18. Dunwiddie TV, Masino SA. The role and regulation of adenosine in the central nervous system. Annu Rev Neurosci. 2001;24:31-55.

19. Fisone G, Borgkvist A, Usiello A. Caffeine as a psychomotor stimulant: mechanism of action. Cell Mol Life Sci. 2004 Apr;61(7-8):85772.

20. Meusel T, Albinus J, Welge-Luessen A, Hahner A, Hummel T. Shortterm effect of caffeine on olfactory function in hyposmic patients. Eur Arch Otorhinolaryngol. 2016 Aug;273(8):2091-5.

21. Kaplan GB, Greenblatt DJ, Ehrenberg BL, Goddard JE, Cotreau MM, Harmatz JS, et al. Dose-dependent pharmacokinetics and psychomotor effects of caffeine in humans. J Clin Pharmacol. 1997 Aug; 37(8):693-703.

22. Hong SC, Yoo YS, Kim ES, Kim SC, Park SH, Kim JK, et al. Development of KVSS test (Korean version of Sniffin' Sticks test). Korean J Otolaryngol-Head Neck Surg. 1999 Jul;42(7):855-60.

23. Cho JH, Jeong YS, Lee YJ, Hong SC, Yoon JH, Kim JK. The Korean version of the Sniffin' Stick (KVSS) test and its validity in comparison with the cross-cultural smell identification test (CC-SIT). Auris Nasus Larynx. 2009 Jun;36(3):280-6.

24. Brickenkamp R, Zillmer EA. The d2 test of attention. Seattle (WA): Hogrefe; 1998.

25. Renda G, Committeri G, Zimarino M, Di Nicola M, Tatasciore A, Ruggieri B, et al. Genetic determinants of cognitive responses to caffeine drinking identified from a double-blind, randomized, controlled trial. Eur Neuropsychopharmacol. 2015 Jun;25(6):798-807.

26. Goel V, Manjunatha S, Pai KM. Effect of red bull energy drink on auditory reaction time and maximal voluntary contraction. Indian J Physiol Pharmacol. 2014 Jan-Mar;58(1):17-21.

27. Mujica-Mota MA, Gasbarrino K, Rappaport JM, Shapiro RS, Daniel SJ.The effect of caffeine on hearing in a guinea pig model of acoustic trauma. Am J Otolaryngol. 2014 Mar-Apr;35(2):99-105.

28. Zawawi F, Bezdjian A, Mujica-Mota M, Rappaport J, Daniel SJ. Association of caffeine and hearing recovery after acoustic overstimulation events in a guinea pig model. JAMA Otolaryngol Head Neck Surg. 2016 Apr;142(4):383-8.

29. Spence C, Kettenmann B, Kobal G, McGlone FP. Selective attention to the chemosensory modality. Percept Psychophys. 2000 Aug;62(6): 1265-71.

30. Spence C, Kettenmann B, Kobal G, McGlone FP. Shared attentional resources for processing visual and chemosensory information. Q J Exp Psychol A. 2001 Aug;54(3):775-83.

31. Hedner M, Larsson M, Arnold N, Zucco GM, Hummel T. Cognitive factors in odor detection, odor discrimination, and odor identification tasks. J Clin Exp Neuropsychol. 2010 Dec;32(10):1062-7.

32. Engen T. The effect of expectation on judgments of odor. Acta Psychol (Amst). 1972 Dec;36(6):450-8.

33. Patel SJ, Bollhoefer AD, Doty RL. Influences of ethanol ingestion on olfactory function in humans. Psychopharmacology (Berl). 2004 Feb;171(4):429-34. 